\title{
PLATFORM WITH CAMERA SYSTEM FOR MEASUREMENT OF COMPENSATORY MOVEMENTS OF SMALL ANIMALS
}

\author{
Patrik Kutilek ${ }^{a} *$, David Skoda ${ }^{a}$, Jan Hybl ${ }^{a}$, Rudolf Cerny ${ }^{b}$, \\ Daniel Frynta ${ }^{c}$, Eva Landova ${ }^{c}$, Petra Frydlova ${ }^{c}$, Aniko Kurali $^{d}$, \\ RADEK DOSKOCIL ${ }^{e}$, VAClaV KRIVANEK ${ }^{e}$ \\ ${ }^{a}$ Czech Technical University in Prague, Faculty of Biomedical Engineering, Kladno, Czech Republic \\ ${ }^{b}$ Second Faculty of Medicine, Charles University, Prague, Czech Republic \\ ${ }^{c}$ Faculty of Science, Charles University, Prague, Czech Republic \\ $d$ „Lendulet“ Evolutionary Ecology Research Group, Plant Protection Institute, MTA Centre for Agricultural \\ Research, Budapest, Hungary \\ ${ }^{e}$ University of Defence, Brno, Faculty of Military Technology, Brno, Czech Republic \\ * corresponding author: kutilek@fbmi.cvut.cz
}

\begin{abstract}
The article introduces systems and methods of a controllable rotational platform used for measuring compensatory movement of small animals. The system, based on a camera subsystem, is located on a mechanical platform powered by a set of three actuators. The subsystems and methods allow to measure angles of the platform's orientation in space and body segment angles in both anatomical and Earth's coordinate systems. The methods of video processing, selection of measurement parameters and detection of anatomical angles are thoroughly described in this article. The study also deals with the software designed in MatLab®, which controls the platform, records and processes videos, and obtains angles for the movement analysis. The system was tested for measuring a head rotation of a small reptile/amphibian and monitored reflective markers on the creature's body by the camera system. This method has never been described before. The new subsystems of the platform and methods for monitoring animal's head compensatory movements can be used in studies of the neural system and its evolution.
\end{abstract}

KEYWORDS: rotational platform; animal; compensatory movement; head; camera; MoCap system.

\section{INTRODUCTION}

The aim to research the process of detection and processing of stimuli for an organism (especially humans) during a change of its position in space stimulated the introduction of the method of dynamic posturography. This method records the body segment movement and is used especially in medical use [1. Examinations using platforms providing shift or tilt of the platform's base with the measured subject. For monitoring the position of selected body segments [2, 3] some more advanced platforms, e.g., the 6DOF2000E (MOOG, East Aurora, NY, USA) are equipped with laser sensors or camera recording systems. These are complex robotic systems with multiple actuators. For the purpose of the evaluation of a movement, dynamic platforms can also provide valuable data for the study of nervous, vestibular, or musculoskeletal systems of animals. Original measurements were carried out with animals tilted in hands and their movement was recorded as shown in the pictures 4 . This method is, however, considered inaccurate. In order to make measurements more effective, special systems, using tilt platforms, have been developed. An example of such typical system is a platform tilting around a set of axis. Even though these systems are usually equipped with multiple electric motors, or they are powered manually, the measurements of the movement are always taken and evaluated during a rotation around a single axis. A typical illustration of the mentioned practice is a study focused on measuring a movement of hares [5]; here, the platform was rotated manually and the markers on the bodies of hares were recorded by cameras. For some experiments with hares, the tilt platforms were additionally equipped with force sensors under the animals, while goniometers were used to measure their movement. The animals are mounted onto platforms with the holders placed on body segments [7], or, if the animals are trained, using only a rough platform surface; this safeguard prevents them from falling, [8]. Some more complex platforms and MoCap systems were used in the studies of nervous system of a domestic cat for an evaluation of its movement in 9, 10. The tilting platform system was, again, equipped with force sensors under the feet of subjects, in this case, however, the electric motor control system recorded not only the momentary values of angles but also the angular velocity. The animal's movements were recorded by Vicon (VICON Motion Systems, Inc, Lake Forest, CA) MoCap camera system. The system, located outside the platform's structure, identified locations of reflective markers on the animal's body automat- 
ically. Some other specific platforms such as those that allow not only rotation but also a translational motion monitoring have also been tested [11. This type of platforms, however, offers a smaller range of rotation; the accuracy of the positional identification depends on the movement of the platform and the MoCap system is located outside the platform. In the case of measuring smaller animals than cats or hares, e.g., rats or frogs, the systems measuring only changes in the body orientation around one axis (i.e., mainly the rotation of the head around the vertical axis) were used. In this case, the movement of the head was examined by means of contrast markers positioned on the head of the monitored animal and a camera placed above the rotation platform [12. The camera was capturing the movement around the horizontal axis 13. However, due to the requirements of a basic research of functioning and evolution of the nervous and vestibular system, there is, nowadays, a need to study segments of smaller animals, such as small amphibians or reptiles of $5 \mathrm{~cm}$ in size. In the past, such devices allowed to measure the movement of smaller mammals only around a single axis [14.

As already demonstrated, recently designed devices with moving platforms allow measuring a movement of mammals of $10 \mathrm{~cm}$ in size, but they are rarely used for measuring smaller animals. All the mentioned works refer to the same disadvantage - the camera system located outside the platform and capturing the animal from rather a great distance (even up to $2 \mathrm{~m}$ ). Therefore, the markers had to be quite large so that they could be seen on the recorded video. If we wanted to measure animals smaller than cats or dogs (like lizards or frogs in our case), we would be limited by downscaling the size of markers and would not be able to get any valid results. The mentioned drawbacks are eliminated by the designed system including a tilt platform and a camera system for measuring the movement of smaller animals. The system also allows a rotation of the platform (with the subject) in all directions, while the camera system mounted on the platform records the movements of body segments in an anatomical coordinate system. The system, manufactured according to the requirements of Faculty of Science of the Charles University in Prague and First Faculty of Medicine of Charles University in Prague [15, will be described, in detail, in this article. To describe and test the functions of the designed system, the head of an animal has been chosen. Although the system was briefly introduced at the conference [15, 16], this article is focused on the description of the subsystematic functions and data processing, that has not been described before.

\section{Methods}

\subsection{SUBSYSTEMS FOR MEASURING MOVEMENT RESPONSE}

To record the movement of an animal on anatomical axes, a special system consisting of several subsystems

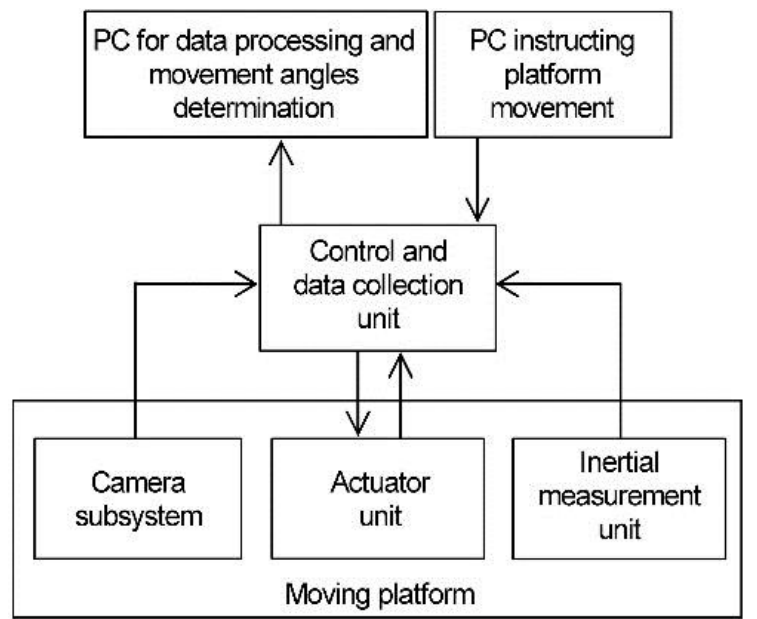

Figure 1. Block diagram of the main subsystems used for measuring a movement response of small animals to the change in orientation of their bodies [15].

has been designed. Figure 1 demonstrates a block diagram of the main subsystems.

The basic part of the system consists of the platform with a camera system, the IMU, and the mounted animal. The platform is tilted by an actuator unit allowing a change of all three Euler's angles. Since the system preforms multiple tasks, the two main tasks (the motor control and camera recording) were allocated to two different PCs, connected via LAN, see Figure 1. The two PCs need to be synchronized, so that the Motor Control PC (MC-PC) becomes aware of Camera Recording PC (CR-PC) and can start storing platform positions, as soon as it starts. The similar synchronized sequence actions happen when the recording stops. We developed this application using MatLab's timers with custom-made file writing and reading functions. The way of the platform's rotation is set by a user on Motor Control PC, from where it is sent to a data collection and control unit located on a stationary base. The base holds the actuator unit connected to the moving platform, see Figures 2 and 3 . The control unit sends the instructions to three actuators and simultaneously obtains information about their position. The IMU and the set of three mutually perpendicular cameras of the camera subsystem give information on the values of the platform tilt angles and angles of the animal's body segment's orientation in an anatomical coordinate system. The IMU and the camera subsystem send information about the platform's orientation and video recording of the animal's body segment to the control unit and the data collection unit. The control and data collection unit is connected to the $\mathrm{PC}$ with a data cable to process and determine the platform yaw, roll and pitch angle values as well as the three anatomical angles of the animal's body segment's orientation.

The designed system (Figure 2 consists of the platform, moving base (the platform plane) made from thin aluminium, creating a space of $200 \times 300 \mathrm{~mm}$ 


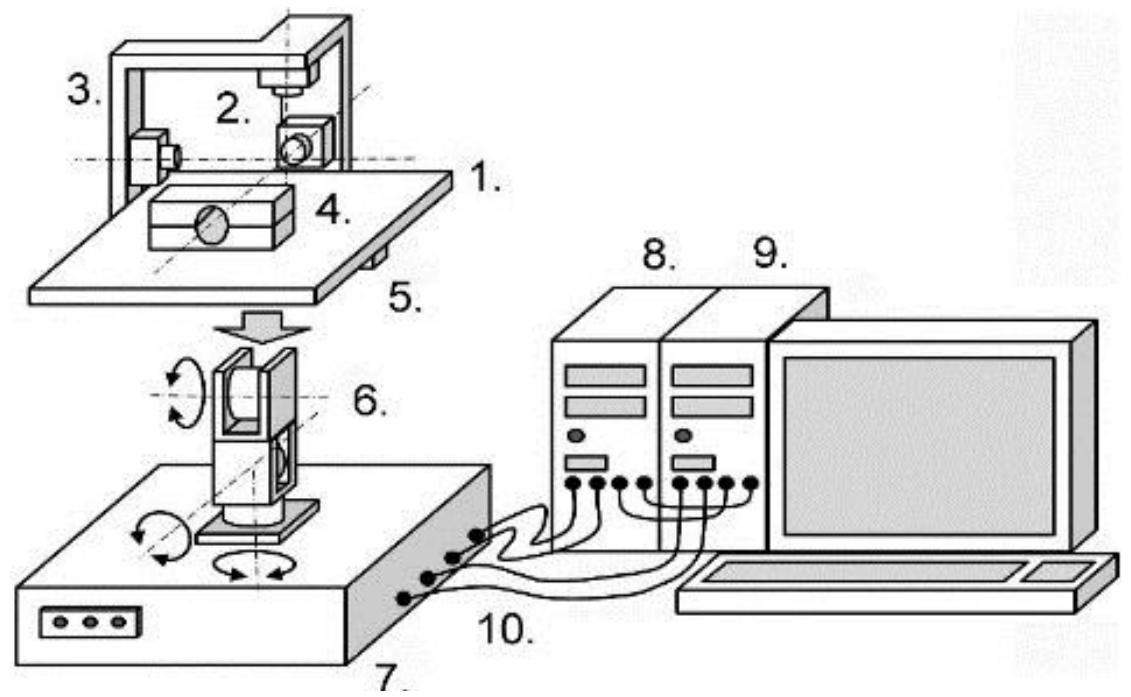

Figure 2. Diagram demonstrating the structure of the system; 1. Moving platform, 2. Camera subsystem, 3. Camera holder, 4. Animal holder, 5. IMU, 6. Actuator unit, 7. Control and data collection unit, 8. PC controlling platform movement, 9. PC for data processing and determination of movement angles, 10. Data cables connecting $\mathrm{PC}$ and Control and data collection units.

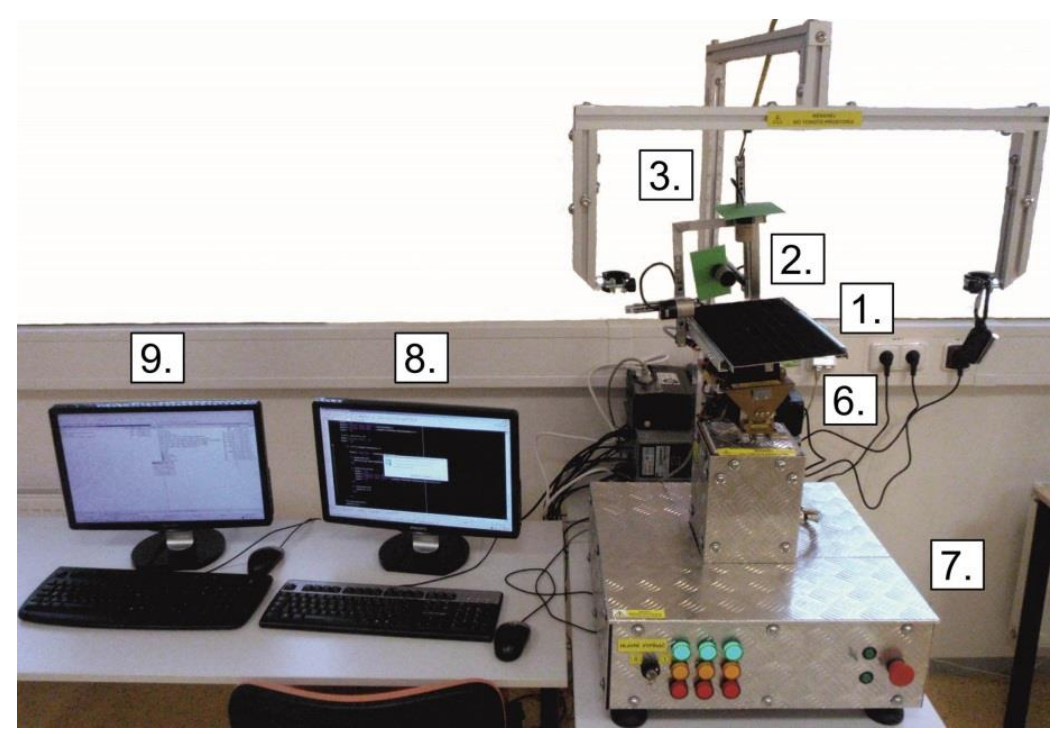

Figure 3. System for measurement of compensatory movements; 1. Moving platform, 2. Camera subsystem, 3. Camera holder, 6. Actuator unit, 7. Control and data collection unit, 8. PC controlling platform movement, 9. PC for data processing and determination of movement angles.

for the placement of the animal. The platform plane allows for manual shift of the animal on the platform's plane perpendicular to the transversal plane. In this way, the animal's head can be set to the focus of the camera subsystem. The solid stationary base, where the control and data collection unit is placed, has a framework made of aluminium profiles. This framework makes space for electric components and a connection point to mount the motors of the actuator unit. The actuator unit consist of three two-phase motors type 60STH88 (Motion Control Products Ltd.) with a basic step of $1.8^{\circ}$ creating a torque of $3 \mathrm{Nm}$. The three motors of the actuator unit are located in mutually perpendicular axes, each of them capable of rotating around a different axis. We used bipolar stepper motors running at 128 microsteps per step, with a magnetic momentum compensation for a steady speed. Microstepping ensures a smooth acceleration/deceleration.

Specific ranges of each rotations have been limited by the software and sensors for each motor individually; this was determined on the basis of a so-called safe operating range depending on the applied current and weight of the platform itself. While the lowest motor can rotate in the range covering $0^{\circ} \pm 170^{\circ}$, both higher motors have been confined to $0^{\circ} \pm 23.4^{\circ}$. The three angles of tilt and rotation were achieved with an accuracy of 1 microstep. The minimum settable 


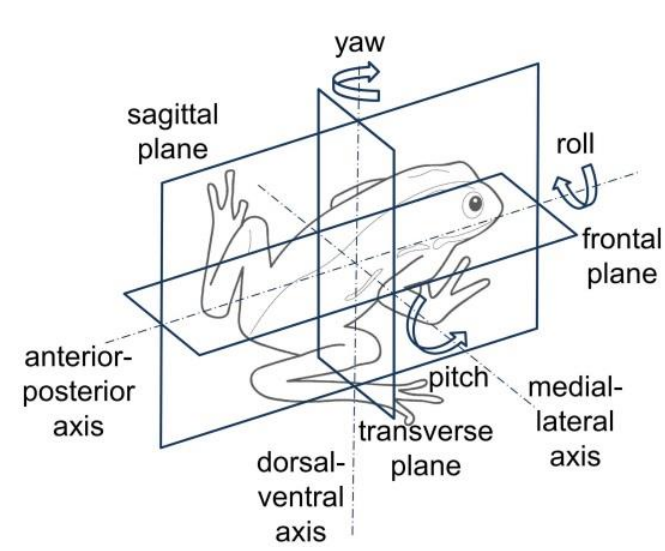

Figure 4. Anatomical coordinate system of the animal and its angular movement in relation to coordinate system related to the direction of gravitational acceleration and Earth's horizontal plane [15].

step was $1.8^{\circ}$ for all three motors, but the sensitivity of the movement is 128 microsteps per $1.8^{\circ}$, giving a movement smoothness of $0.014^{\circ}$. The reason why we did not let the user execute movements smoother than $1.8^{\circ}$ is that the motors cannot be stopped anywhere between. Every time they stop, the finishing stroke is present within a one second delay setting the platform's angle to the closest stable point (every $1.8^{\circ}$ away). Also, the $1.8^{\circ}$ step is inevitable for accelerating/decelerating the motion of the moving platform.

The Calibration and checking of the tilt and rotation angles is performed by the control and data collection unit obtaining information from the IMU. This combines a three-axis gyroscope, three-axis accelerometer, 16 bit ADC and a digital movement processor for an accurate monitoring of fast and slow movements in gyroscope ranges of $\pm 250^{\circ} / \mathrm{s}$ as well as in accelerometer ranges of $\pm 4 \mathrm{~g}$. Ranges of angular movements of the three motors are limited to avoid coincidence between the platform and the base construction. The limit of the range of the motor movement is set to $46.8^{\circ}$, which is a higher value than in similar experiments [7, 8]. A light tin holder with rails for the cameras of the subsystem and another one to hold the animal are fitted onto the platform. Cameras are placed so that they are perpendicular to each other; each records one of the body planes of the animal. We used Defender G-lens 2577 HD720P cameras with a high definition of $720 \mathrm{p}, 10 \times$ digital zoom and a suitable focus distance starting at $3 \mathrm{~cm}$. The resolution was set to $320 \times 240$ pixels to speed up the data saving process and maximize the number of captured frames per a measurement. The camera's angle of view was $56^{\circ}$, which enabled a scope of a significant part of a small animal in a particular anatomical plane. Tests have proven that the geometrical distortion of the view was negligible, taking into consideration the resolution, the distance from the object and the size of markers. It was also proven that the distortion does

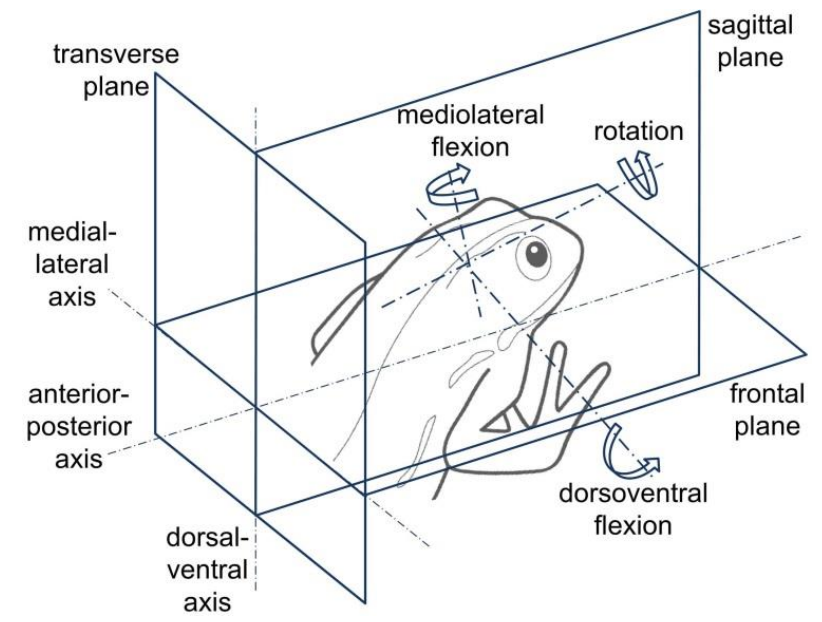

FiguRE 5. Movement of body segment (head) in anatomical coordinate system of an animal.

not affect the angle calculation. We can also regulate the amount of light coming from the LED strip lights placed on the camera holder. They influence the maximum achievable framerates of the used cameras and enable to set the light conditions so that the markers are as contrasting as possible, which facilitates their recognition in the captured frames.

As mentioned before - lighting of the scene has a huge impact on frames per second (fps). The general rule is: more light means more fps. The demand for a higher resolution results in lowering the final fps, which actually means that we have to find a compromise. We have discovered that the resolution of $320 \times 240$ pixels is optimal for our applications. Such resolution would normally allow up to $60 \mathrm{fps}$. The lighting of the scene has to be set so that markers are not over-exposed.

\subsection{MEASUREMENT OF BODY SEGMENT MOVEMENT OF SMALL ANIMALS IN 3-D SPACE}

The platform allows a rotational motion of the animal's body in $3-\mathrm{D}$ space. The initial position was a horizontal plane and a direction of a gravitational acceleration. The animal was mounted to the platform in a way ensuring that the three anatomical axes were in accordance with the three main axes of the platform (Figure 4). The change in the animal's orientation is defined by Euler's angles - yaw, pitch and roll, Figure 4

To determine the orientation of the body segment in a coordinate system, it is necessary (in the case of the head) to define three movement angles in the anatomical system of the animal's body, which match the coordinate system of the moving platform. They are - mediolateral flexion (i.e., rotation about mediolateral axis), dorsoventral flexion (i.e., rotation about dorsoventral axis) and the head rotation (i.e., rotation about anterior-posterior or say rostro-caudal axis), Figure 5 . 


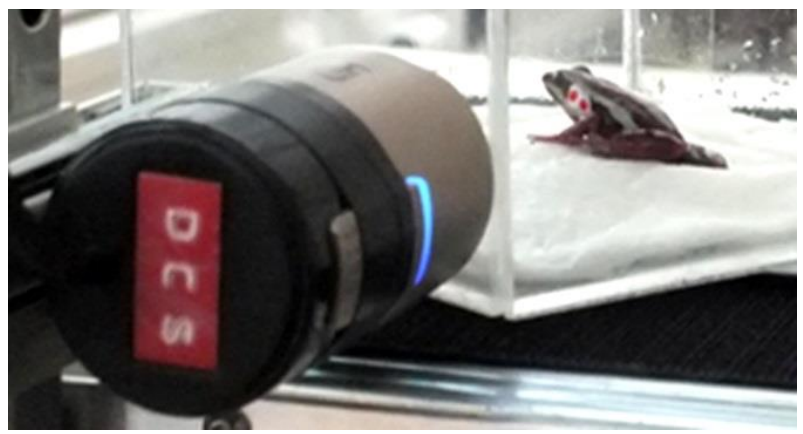

Figure 6. Placement of the marked animal inside the box on the platform.

These angles can be measured by appropriately placed markers on the animal's head. Cameras of the camera subsystem are located in a way ensuring their optical axes are perpendicular to each other, and at the same time perpendicular to sagittal, transversal and frontal axes of the animal placed on the platform [15. Markers can have a form of reflective plastic stickers [9] or their paper alternative [5]. It is, however, their disadvantage that they are rather big [9], therefore, anatomically harmless paint of a contrastive colour was applied on the animal skin instead. Two spots were made for each plane. It had to be ensured they do not coincide with each other and their placement on anatomically fitting parts does not coincide with minimal intrapopulation variability.

As for the placement of the animal onto the platform — we needed a solution, which would give animals more freedom to move, but not a chance to escape. For larger animals (about $10 \mathrm{~cm}$ length of body), Velcro straps were found as the most suitable, but smaller animals (less than $10 \mathrm{~cm}$ length of body), were placed into transparent closable boxes equipped with velcro at the bottom, see Figure 6. If the subject turned its segments inside the box, the measurement was cancelled.

\subsection{Controlling of the Platform AND DATA RECORDING}

Graphical Users Interface (GUI) has been developed to control the movements of the platform and to record and process videos in MatLab (MatLab R2010b, Mathworks, Inc., Natick, MA, USA). The user can modify the angle step $\left({ }^{\circ}\right)$, speed of rotation $(\% / s)$ and the direction of the movement of each motor. A GUI for recording allows the user to determine the fps. In the case of camera's settings, for any correct calculation of anatomical angles, it is suitable to use a higher frequency rate for the record [12. In one case [5], a 25 fps rate was used, however, in [9, 11, even $120 \mathrm{fps}$ rate was used. This means that, even before making the final decision, some expected movements of a body segment should be taken into consideration. Our lowest framerate usually varies based on the light conditions during the measurement, but roughly equals to $30 \mathrm{fps}$

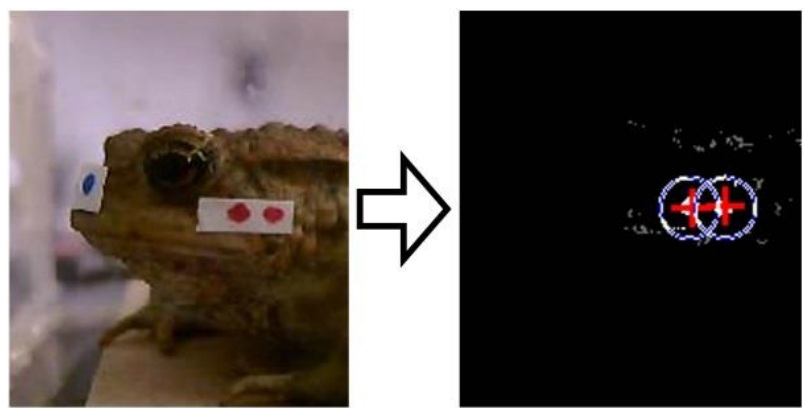

Figure 7. Demonstration of how to work with a border-radius close-up. The pixels taken into consideration are white, red crosses mark detected centers and the pixels ouside the range of a close-up (highlighted with circles) are in grey zone.

due to the recording slow compensation movements of reptiles.

A button for post-processing has been added to the GUI. What the algorithm does, has been partially described in the previous section. First, it cuts off redundant frames that are recorded before MatLab manages to shut all three cameras or before it starts recording. To determine, how many frames has the cameras recorded, we used MatLabs's ,getdata“ function, which can return timestamps. Timestamps tell us exactly when each frame was recorded. At the end of each measurement, everything is stored in a text file, including the name and fps of each video, as the processing of the videos is very time consuming.

Last part of the GUI enables the user to calculate angles automatically. It also has a sub-part, where a user can play videos and see what the software has detected, or how angles are changing in time.

\subsection{VIDEO PROCESSING}

Marker coordinates are detected in the pictures automatically using the contrast between their own colour and the colour of the animal's skin. This phenomenon is used in the calculation software created in MatLab. The detection of markers is based on the pre-set colour tolerance on the scale from 0 to 255 for all three colours in the RGB spectrum, converting each pixel to a binary mask. The tolerance is pre-set for each colour and can be adjusted by a user to any value ranging from 1 to 50. It means, for example, that any pixels found suitable for the selected colour with the value of \pm 50 will be marked on the frame as detected. The tolerance is common for all three colours in the RGB spectrum and cannot be modified separately. Cropping a border-radius (Figure 7) is another useful technique. Sometimes, we can detect pixels in the background of the scene that are the same colour as markers, or are within the tolerance range [18]. Those pixels are usually fairly far away from those indicating the markers 19. Therefore, after we find the approximate centres of the markers on the previous frame using $k$-means, we perform a deletion scan of any detected pixels within the specified radius from 


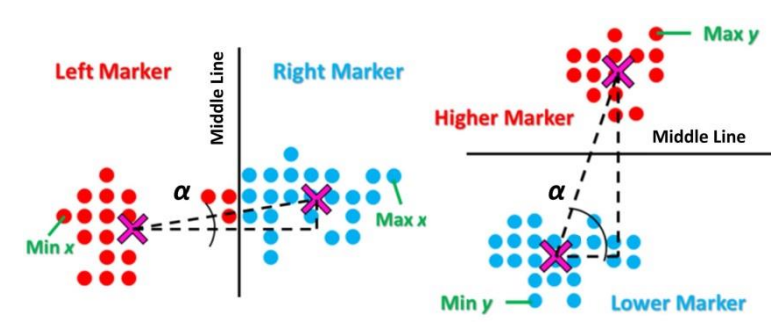

Figure 8. Method of slicing the screen. Crosses marks are computed centres of markers and $\alpha$ is the detected angle.

each previous centre on the current frame and process the frame again in order to receive information from pixels near the centres of the previous frame.

One approach to the detection of markers' centres is to make a MatLab's algorithm of $k$-means detect two centres in relation to white coloured pixels, as the input arguments. The $k$-means function, implemented in MatLab software [20, is very accurate (mistakes are very rare) and comfortable, however, also timeconsuming. The new method first detects all the pixels complying with the requirements of the tolerance and cropping of radius conditions, as was described above. Then, it checks the $x$ and $y$ coordinates of every detected pixel and based on that determines whether pixels are located above each other ( vertically in the image), or next to each other (horizontally in the image). The algorithm then finds the central line between bordering pixels. For example, if the pixels are horizontally situated on the slide, we take the smallest and the biggest $x$ coordinate of the detected pixels and the vertical central line is right between them. Determination of the centre is given by

$$
C_{x}=\frac{\sum_{i=1}^{N} x_{i} A}{\sum_{i=1}^{N} A}, \quad C_{y}=\frac{\sum_{i=1}^{N} y_{i} A}{\sum_{i=1}^{N} A},
$$

where $C$ stands for centres and its index marks the plane for which we count it. The number of detected pixels is $N$ and $A$ is the area of the detected pixel. Using this simplification, we get an equation for the mean value of the $x$ and $y$ coordinates. To be even more accurate in the estimation of the centre positions, we can completely replace the equation by a median calculus. After both centres on both sides of the central line are detected, the method checks the distance of all detected pixels from previous centre locations using a classical Pythagoras's theorem. The pixels, which are further than the specified cropped radius value, are excluded from the previous centre positions and are stained grey in the preview image. Then, the method checks the medians again, using only remaining pixels, to get more precise centres' locations.

This method is much faster than the $k$-means algorithm, unfortunately, it is not entirely error-proof.
The sharp division along just two dimensions (vertically, or horizontally) can be inaccurate. For example, when markers get close to each other and position themselves diagonally, the division line can cut one of the markers and take some of its pixels as pixels of the other marker (Figure 8 - slowly shifting the detected centres' locations, which results in losing one of the markers. The situation is optimal when the division line is made at a specific angle to clearly separate both markers. Implementation of such algorithm would, however, be too time consuming, which we were trying to avoid. Based on the above, we decided to conduct a $k$-means check after the set of every 50 frames to prevent losing the marker for a longer period of time. We also needed to check whether the intercentral distance has not changed by more than $15 \%$ of its length on the previous frame, in which case $k$-means are launched again automatically to correct the error. This is particularly convenient in the case of the wrong estimation, which may happen, although very rarely, as was mentioned before.

Last but not least, we had to deal with a slight changes in the colour of the marker due to the changes of the light conditions. If we rely on detecting only one colour with a specified tolerance throughout the whole video, we may as well end up with only few applicable frames and rest would have to be discarded. Therefore, we had to implement a method based on a vector containing all the information about the colour, which the user wants to detect, such as tolerance and cropping radius of each frame where the mentioned values were changed. The user can then save the vector for each video to a txt file and return to it later if necessary. At the beginning, the user also has to indicate that he wishes to use the keyframe vector and click on the colour manual selection button. The preview of the first frame appears along with the preview of the detection. The user then clicks on one of the markers he wishes to detect in the preview, adjusts the tolerance or cropping radius range if needed, and he can then use the slider to scroll to the other frame. Once he notices the marker is diminishing on the detection preview, he can refresh the detection colour by another click on the marker. The values are stored within a vector and from then on, the software is detecting the last settings until the end of the video, or until another change is made and another column appears in the vector.

There is another useful feature that can be implemented in preliminary video processing tests. Since the markers change their colour quite often, an Adaptive Color Change algorithm (ACC algorithm) was implemented. The basic idea is to observe how the colour of the markers change in the course of time and adjust changing the detection colour in the settings automatically, so that the user does not have to do it manually. Its mainframe works similarly to the keyframe vector described above, but unlike the keyframe, the ACC vector stores the information 
about colours, tolerance and cropping radius for every frame of the video, not just keyframes specified by the user. The keyframe vector is, however, superior to the ACC vector, because the ACC algorithm does not try to change the values belonging to the keyframe, but rather uses them instead. The software always tries to use the detection colours specified by the previous frame and see what pixels it detects. Then, it extracts the information about the RGB spectrums of the detected pixels on the current frame and calculates a median of each of the three values. The median values are implemented into the ACC vector and then serve as the detection colour for the current frame. The detection is launched once more with updated values and centres are located for the needs of the cropping radius method. Then it shifts to another frame and repeats the process described above until the required or the last frame of the video is reached.

\subsection{Method FOR DETERMINATION OF ANATOMICAL ANGLES OF MOVEMENT}

To measure the position of particular segments of an animal (in our case, the head), the platform is equipped with a camera system and the IMU. To determine the anatomical angles, it is necessary to measure using Euler's angles representing changes in angular positions of the platform in space. For this reason, it is essential to calibrate the system prior to each measurement. The platform's coordinate system (i.e., platform orientation in 3-D space) is set in an accordance with gravitational acceleration and horizontal plane of the Earth on the basis of the IMU data before each measurement. In order to secure this, the IMU was mounted to the platform during its assembly in such a way that the three main axes of the IMU are parallel to the main platform axes. After the calibration, the pitch, yaw and roll angles are measured too and the information obtained from the three motors verifies the accuracy of the reached pitch, yaw and roll angles.

The camera holder is mounted onto the platform holding three cameras (set so that the three axes of the cameras are mutually perpendicular and also parallel with the three main axes of the platform) and it enables to record the movement of the points in planes respective to planes perpendicular to the main axes of the platform. If the animal's body is placed on the platform correctly, it is possible to record the movement of segments in anatomical planes of the body, i.e., sagittal, frontal and transversal. The animal must be fixed in a way that the three anatomical axes are parallel to the three axes of the platform. If we, however, focus only on measuring the evolution of the angle value change, the accurate fixing of animal does not seem to be so crucial.

Determination of the segment rotation on a particular plane is given by the position of two markers on a body segment (i.e., anatomical points) and the camera setting. Let us suppose the angle calculation is defined by two markers placed on the head and physical axis defined by the tilt of a camera, while the camera is parallel with the main axis of the platform. The cameras (during the assembly process) are set in a way that their picture matches a particular plane and also two axes of a picture (i.e., physical axis of the picture - vertical and horizontal), which are parallel with the main axes of the platform. Figure 8 demonstrates the example of two captured markers on a segment (e.g., head) to calculate mediolateral flexion, or, angular movements in the frontal plane. The angle between the anatomical axis and the horizontal picture is an angle between the $\mathrm{v}$ vector given by a camera setting in an accordance with the platform axes and the $u$ vector representing the coordinates of points determined in the picture. The angle between the vectors is given by

$$
\alpha=\arccos \frac{u \cdot v}{|u||v|},
$$

where $u=\left(a_{x}-b_{x}, a_{y}-b_{y}\right), v=(1,0), a_{x}, b_{x}$ are coordinates of two points/markers on the $x$ axis, and $a_{y}, b_{y}$ are coordinates of two points/markers on the $y$ axis of a picture from one of the cameras [21, 22]. This calculation is utilized when calculating movement angles in all three anatomical planes, or three pictures of a simultaneous record from cameras in the subsystem. Then, the records from cameras, i.e., anatomical angles, are coupled with data, i.e., angles of the rotation, of the motors and the IMU. Thus, body segment angles in space for its respective planes are calculated by a mere sum or difference between the anatomical angle and the platform movement angle.

If it is not possible to determine the value of angles due to a short temporary loss of information on marker positions, a data interpolation method is implemented into the software. The interpolation method allows to calculate the missing kinematics data within a time series of angles. For this purpose, the Cubic spline data interpolation, implemented in MatLab software, was used 23 .

\section{EXPERIMENTS}

In our measurements, we used frogs and geckos. We were mostly working with Common Toads (Bufo Bufo), and Leopard Geckos (Eublepharis Macularis), but we also measured some other frog specimen of various sizes. The animals were specifically selected for a systematic testing. We focused on the measurements of the relative movement of the head in relation to trunk. The body of the subject was placed in such a way that the longitudinal axis corresponded with the longitudinal axis of the platform. Relative angles of the head movement in relation to the platform were determined by markers placed on the upper part of the head, two on the front and two on the side part of the head. The animal was placed onto the platform, and a gradual and sequenced rotation was carried out. 


\subsection{Selection of MEASUREMENT CONDITIONS}

To test and verify the designed system and method, the rotation of the animal around only one axis, similarly to several other studies of animal body segment movement [7, 8, was chosen. The animal body can be rotated in transversal anatomical plane, i.e., by change in the roll angle of the platform [5] 8 . Some studies, however, also describe the movement on sagittal plane; i.e., change in the pitch angle of the platform [7. 9 . We primarily focused on the head movement in the sagittal plane and controlled the change of orientation in the pitch angle of the platform, where the most significant compensatory movements of the head can be expected. As for the selection of the measurement conditions, the platform can be moved periodically by various types of the course of the angular velocity, e.g., by the sinus course of angles (with a frequency of 0.5 or $1 \mathrm{~Hz}$ and an amplitude of $\pm 20^{\circ} / \mathrm{s}$ ) or linear course (between $\pm 20^{\circ}$ ), so called ramp-and-hold mode, as used in [5]. In the research of animal movement, the sinus course is used less often than the linear course. The sinus course (with a frequency of $1 \mathrm{~Hz}$ ) was used and also in [7, 13] (with a frequency of $0.25-$ $5.5 \mathrm{~Hz}$ and an acceleration of $\left.100^{\circ} / \mathrm{s}^{2}\right)$. In [16, 24], the linear course was used along with a tilt range of $\pm 20^{\circ}$. Tilts are, therefore, usually symmetric in relation to the initial horizontal position of the platform. Maximum values of tilt angles can reach up to $40^{\circ}$ in healthy subjects, in the case of roll and pitch angles [7, 8. During an evaluation of pitch angles, even $+90^{\circ} /-90^{\circ}$ angle was used, however, the movement of subjects was carried out manually and the movement record step was $20^{\circ}$, instead of electronically powered platform [4. In some studies of animals (e.g., cats), we may identify lower angles, such as $6^{\circ},[9,11$, or $\pm 1^{\circ}$ to $\pm 20^{\circ}$ [13]. Lower values (from $3^{\circ}$ to $7^{\circ}$ ) are, however, used rather in cases of postural stability in humans, due to a higher centre of mass position compared to quadripedal animals, where the base support is larger with a lesser risk of losing the balance in higher tilt values [25-27]. Initial or final phase of the linear change can be defined by the maximum reached angle value or setting the period of an angular movement of the platform [7, 8. Certain values of angular velocities then correspond to such movement. An angular velocity of $40^{\circ} / \mathrm{s}$ was used in [5, 9, 11, while in [7, the values were higher; e.g., $50^{\circ} / \mathrm{s}$. These values are comparable with those used in studies of humans, $36^{\circ} / \mathrm{s}$ to $50^{\circ} / \mathrm{s}$ [25]27. Since the mentioned cases discuss dynamic effects, (high or low), the velocity was high enough to reliably cause an automatic postural response $(\mathrm{APR})$ in the subjects [28]. The total measurement time lasted only few seconds, e.g., $\max 3 \mathrm{~s}$ [9] per one noncyclic movement. We select the movement angles for the preliminary testing on a greater scale, ranging between $+23^{\circ} /-23^{\circ}$, as the design allows.

Referring to the above-mentioned, we selected move- ment angles for the preliminary testing on a greater scale, ranging between $+23^{\circ} /-23^{\circ}$, according to what the design of the construction allows. As for the selection of angular velocity, it did not fall within the objectives of the research commissioned by Faculty of Sciences of the Charles University in Prague and First Faculty of Medicine of Charles University in Prague. In both these institutions, the research has been, so far, primarily focused on movement responses of small reptiles with the nervous system on a lower evolutionary level than that in mammals. Using a high angular velocity is not appropriate with small reptiles.

In the case of fast movements of body segments, we may encounter a problem generally known as the D'Alembert's inertial force and a moment of a force. These forces and moments would have to be compensated by the forces provided by the muscular system of the animal. For this reason, at a high angular velocity, change in the orientation of the body segment relative to the vector of gravity is not primarily compensated . Therefore, the angular velocity of $2.5^{\circ} / \mathrm{s}$ and $5^{\circ} / \mathrm{s}$ was selected for the purpose of studying the movement responses. With each speed, the platforms always move from one side to the other and back, repeating multiple times to ensure useful data. Finally, we tried to use a "sine wave" mode [5], which is actually a negative cosine wave. In this regime, the platform starts at one end and slowly accelerates up to the peak speed reached in the neutral position, and then decelerates until it closes the cycle at the other end. The rotation speed profile resembles a negative cosine wave, hence the name.

\subsection{Results}

The system provides information on the flexion angles (dorsoventral and mediolateral) and the rotation angle. The example of dorsal flexion of the head of an unstressed animal is demonstrated in Figure 2, Based on the information about the relative head angles in relation to the platform and angles of the platform movement in Earth's coordinate system (i.e., platform pitch angle), it is possible to calculate the head movement in relation to the Earth's horizontal line [29], i.e., the head pitch angle, Figure 9

\section{Discussion}

A special device with a moving platform was constructed to measure body segments of small animals. The system is particularly suitable to carry out a precise measuring of a body response to changes in the body orientation. A record - the output of the system - is supposed to contribute to a further scientific research of the evolution of segment's anatomical angle values and segment angles in relation to the horizontal plane of Earth. In the tests of the designed device, we measured the movement of a head during changes in orientation of the whole body. We discovered that in the case of platform moving with the toads, the subjects perform a compensating head 


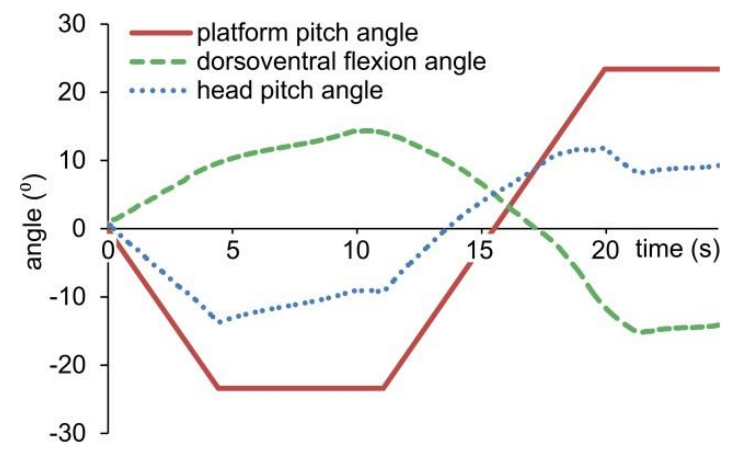

Figure 9. An example of a diagram measuring head movements of the Common toad in the sagittal plane of its body.

movement in almost all the cases. The range and extent of the compensation will be used in the future research. Nonetheless, the stress definitely seems to be a relevant factor, as some frogs just got inflated and refused to move, while others relaxed and sat, compensating for even the slightest movement. In the case of unstressed animals, preliminarily findings show that the compensation of a platform movement has a certain inertia after the completion of the platform movement, as shown in Figure 9 Finding the reasons for this behaviour of animals and its quantitative description, will be a part of the follow-up research.

To compare the designed system with some others, we can observe that, for example, in the case of [5], only manual positioning and a lower video frequency was used, while our system uses 30 fps. Another shortcoming of the [5] system is that the recordings were processed manually, i.e., markers were made manually in the pictures. The software that we created enables detection of contrast markers automatically, which speeds up the processing of the record. Another advantage of the outcomes of our study is that since the skin of our subjects is painted, sticker markers are not used, which, again, facilitates experiments and makes them cheaper. Our system also avoids difficulties in measuring very small animals (under $10 \mathrm{~cm}$ of length) by mounting them directly onto the platform. Instead of mechanical sensors for monitoring angles used in $[6$, our system is contactless, non-invasive and more suitable for measuring the 3-D movement. Compared to MoCap systems used for measuring animal movements based on the electromagnetic principle [13, 30, for example Fastrak (Polhemus, Colchester, VT), the accuracy of our system is affected by electronic parts such as actuator unit motors.

The method we designed is based on a single axis rotation in accordance with [5, 6] and is adjusted to measure slow movements of reptiles/amphibians. Measurements of the head movement of smaller reptiles/amphibians on a rotating platform with the mounted camera system are original and have not been used so far. Although some recordings of mammal head movements were mentioned, for example, in [11, 30, their system's construction and measuring methods did not allow measuring of relative and absolute values of the head movement angles in all three anatomical planes. Further research into the movement might deal with the issue of a cyclic repetition of transition between stationary (or extreme) platform positions, which lasts just a few seconds, like in [7, 8, while the cyclic movement is not limited by the number of cycles. The measuring also allows a repetition of identical tests under the same conditions and a collection of long-term data. [9]. The system can be used for measuring the movement of animals trained to track targets, as stated in 30 . Based on the above, it is obvious that the platform and methods for monitoring animal's head compensatory movements can be used in non-contact studies of body movement influenced by functioning of the nervous system and its evolution, as described in detail in the submitted research [4-14]. The measurement conditions (i.e., the measurement methodology for the study of the nervous system) may be the same as in [4, 9, 14] or in an accordance with the suggestions in this article.

\section{Conclusions}

The article presents a new system and methods for measuring the movement response of small animals to changes in their body orientation. The method is based on a camera system located on a moving platform and powered by three actuators. The system was tested on measuring the head rotation of small reptiles/amphibians. The results also include a presentation of the method, which measures compensation movements of animals. The new system intends to measure movements of small animals within the scope of veterinary and scientific usage and allows to diagnose pathological symptoms in the animal's posture. Further testing of systems for the evaluation of some more complex movements is expected within a follow-up research.

\section{ACKNOWLEDGEMENTS}

This work was done in the framework of project SGS16/109/OHK4/1T/17. The authors would also like to thank Progredior Kybernetes Ltd. for the manufacturing of the electronically powered platform.

\section{REFERENCES}

[1] Teszler, C. B., et al. Sonovestibular symptoms evaluated by computed dynamic posturography. The international tinnitus journal, 6(2), 140-153, 2000.

[2] Luu, B. L., et al. "Validation of a robotic balance system for investigations in the control of human standing balance." IEEE Transactions on Neural Systems and Rehabilitation Engineering 19.4: 382-390, 2011. DOI:10.1109/TNSRE.2011.2140332

[3] Tsai, Y. C., Hsieh, L. F., and Yang, S. "Age-related changes in posture response under a continuous and unexpected perturbation." Journal of biomechanics. 47.2: 482-490, 2014. DOI:10.1016/j.jbiomech.2013.10.047 
[4] Heath, J. E., Northcutt, R. G, and Barber, R. P. "Rotational optokinesis in reptiles and its bearing on pupillary shape." Journal of Comparative Physiology A: Neuroethology, Sensory, Neural, and Behavioral Physiology 62.1: 75-85, 1969. DOI:10.1007/BF00298043

[5] Beloozerova, Irina N., et al. "Postural control in the rabbit maintaining balance on the tilting platform." Journal of neurophysiology 90.6: 3783-3793, 2003. DOI:10.1152/jn.00590.2003

[6] Hsu, L. J., et al. "Effects of galvanic vestibular stimulation on postural limb reflexes and neurons of spinal postural network." Journal of neurophysiology 108.1: 300-313, 2012. DOI:10.1152/jn.00041.2012

[7] Lyalka, V. F., et al. "Impairment and recovery of postural control in rabbits with spinal cord lesions." Journal of neurophysiology 94.6: 3677-3690, 2005. DOI:10.1152/jn.00538.2005

[8] Lyalka, V. F., et al. "Impairment of postural control in rabbits with extensive spinal lesions." Journal of neurophysiology 101.4: 1932-1940, 2009. DOI:10.1152/jn.00009.2008

[9] Macpherson, J. M., et al. "Bilateral vestibular loss in cats leads to active destabilization of balance during pitch and roll rotations of the support surface." Journal of neurophysiology 97.6: 4357-4367, 2007. DOI:10.1152/jn.01338.2006

[10] Deliagina, Tatiana G., et al. "Neural bases of postural control." Physiology 21.3: 216-225, 2006. DOI:10.1152/physiol.00001.2006

[11] Ting, L. H., Macpherson, J. M. "Ratio of shear to load ground-reaction force may underlie the directional tuning of the automatic postural response to rotation and translation." Journal of Neurophysiology 92.2: 808-823, 2004. DOI:10.1152/jn.00773.2003

[12] Shinder, Michael E., and Jeffrey S. Taube. "Active and passive movement are encoded equally by head direction cells in the anterodorsal thalamus." Journal of neurophysiology 106.2: 788-800, 2011. DOI:10.1152/jn.01098.2010

[13] Dieringer, N., and Precht, W. "Compensatory head and eye movements in the frog and their contribution to stabilization of gaze." Experimental brain research 47.3: 394-406, 1982. DOI:10.1007/BF00239357

[14] Dieringer, N., Cochran, S. L., and Precht, W. "Differences in the central organization of gaze stabilizing reflexes between frog and turtle." Journal of Comparative Physiology A: Neuroethology, Sensory, Neural, and Behavioral Physiology 153.4: 495-508, 1983. DOI:10.1007/BF00612604

[15] Kutilek, P., et al. "System for measuring movement response of small animals to changes in their orientation." Applied Electronics (AE), 2015 International Conference on. IEEE, 2015.

[16] Hybl, J., et al. "Methods for evaluation of kinematic motion data of animal's body on dynamic platform." Mechatronics-Mechatronika (ME), 2016 17th International Conference on. IEEE, 2016.
[17] Simsik, D., et al. "The video analysis utilization in rehabilitation for moblity development." Lékař a technika. Česká republika: 4-5, 2004.

[18] de La Bourdonnaye, A., et al. "Practical experience with distance measurement based on single visual camera." Advances in Military Technology 7.2: 49-56, 2012 .

[19] Doskocil, R., Krivanek, V., and Stefek, A. "Contribution to determination of target angular position by single visual camera at indoor close environs." Mechatronics 2013. Springer, Cham. 379-385, 2014.

[20] Gonzalez, R. C., Woods, R. E. Eddins, S. L. Digital Image Processing Using MATLAB, 2003. ISBN-13: 978-0130085191, ISBN-10: 0130085197

[21] Kutilek, P., J. Charfreitag, and Hozman, J. "Comparison of methods of measurement of head position in neurological practice." XII Mediterranean Conference on Medical and Biological Engineering and Computing 2010. Springer Berlin Heidelberg, 2010. DOI:10.1007/978-3-642-13039-7_114

[22] Kutílek, P., and Hozman, J. "Determining the position of head and shoulders in neurological practice with the use of cameras." Acta Polytechnica 51.3, 2011.

[23] Hazewinkel, M. "Spline interpolation", Encyclopedia of Mathematics, Springer, 1994. ISBN 978-1-55608-010-4

[24] Dieringer, N. "The role of compensatory eye and head movements for gaze stabilization in the unrestrained frog." Brain research 404.1: 33-38, 1987. DOI:10.1016/0006-8993(87)91352-7

[25] Allum, J. H. J., and Pfaltz C. R. "Visual and vestibular contributions to pitch sway stabilization in the ankle muscles of normals and patients with bilateral peripheral vestibular deficits." Experimental Brain Research 58.1: 82-94, 1985. DOI:10.1007/BF00238956

[26] Carpenter, M., Allum, J., and Honegger, F. "Vestibular influences on human postural control in combinations of pitch and roll planes reveal differences in spatiotemporal processing." Experimental brain research 140.1: 95-111, 2001. DOI:10.1007/s002210100802

[27] Nardone, A., T. Corra, and M. Schieppati. "Different activations of the soleus and gastrocnemii muscles in response to various types of stance perturbation in man." Experimental brain research 80.2: 323-332, 1990. DOI:10.1007/BF00228159

[28] Diener, H. C., et al. "Early stabilization of human posture after a sudden disturbance: influence of rate and amplitude of displacement." Experimental Brain Research 56.1: 126-134, 1984. DOI:10.1007/BF00237448

[29] Haque, A., and Dickman, J. D. "Vestibular gaze stabilization: different behavioral strategies for arboreal and terrestrial avians." Journal of neurophysiology 93.3: 1165-1173., 2005. DOI:10.1152/jn.00966.2004

[30] Stapley, Paul J., et al. "Bilateral vestibular loss leads to active destabilization of balance during voluntary head turns in the standing cat." Journal of neurophysiology 95.6: 3783-3797, 2006. DOI:10.1152/jn.00034.2006 